\title{
Analysis of a Signal Preprocessing Method for Blade Tip-Timing without the Once-per Revolution Sensor
}

\author{
Jilong Zhang, Fajie Duan* and Jiajia Jiang \\ Tianjin University, Nankai, Tianjin 300072 China \\ *Corresponding author
}

\begin{abstract}
Blade tip-timing is an effective method for blade vibration online measurement of turbomachinery. The majority of tip-timing systems require a once-per revolution (OPR) sensor installed close to the shaft to generate timing reference. In this article a tip-timing signal preprocessing method which requires no OPR sensor is analyzed. The method presented uses only tiptiming signal to obtain the vibration information of the blade and the rotating speed of the rotor. Error analysis indicates the method is less sensitive to the bend and torsional vibration of the shaft and the rotating speed measurement error can be limited. The measurement error of vibration displacement difference is analyzed and compared with the method which requires the OPR sensor. Experiments are conducted and the results indicate that the method is feasible for BTT signal preprocess without the OPR sensor and has good accuracy.
\end{abstract}

Keywords-blade vibration measurement; blade tip-timing; onceper revolution

\section{INTRODUCTION}

Blades are the key components of large rotating machinery and the vibration parameter measurement of blades under working condition is crucial for the analysis of blade dynamical behavior, damage and failure mechanism and life estimation [1]-[3].The blade tip-timing (BTT) method has been widely used in blade vibration measurement for its advantage of noncontact and ability to measure the vibration of all blades in realtime. The BTT system measures the time when a blade tip arrives at the tip-timing sensor, and the time of arrival (TOA) changes because of the blade vibration. The tip-timing data is converted to the vibration displacement, and parameters such as vibration frequency, amplitude and modal can be analyzed through the identification algorithm[4]-[6]. The TOA can be acquired by different kinds of sensor such as optical fiber sensor, capacitive sensor, magnetoresistive sensor and microwave sensor[7]-[10].

The BTT system requires a once-per revolution(OPR) sensor to generate a timing reference for each revolution of the rotor, and the OPR signal is also used to measure the rotating speed. The sensor is typically mounted near the shaft and output the OPR signal when detecting a mark on the shaft. The OPR signal is affected by the shaft bend and torsional vibration, and all these noises are introduced to the BTT measurement. The OPR sensor is difficult to install in some cases, such as high-pressure compressor of a dual-rotor engine, of which the shaft is isolated from the axis of the low-pressure compressor. The BTT system will fail to analyze the vibration when the
OPR signal loses, although the tip-timing data is acquired correctly.

In this article, the measurement error of typical preprocess method which requires OPR sensor is analyzed first, which includes the influential factors and disadvantages. A signal preprocessing method for Blade tip-timing without the onceper revolution sensor is analyzed. The tip-timing data is converted to the vibration displacement difference between neighboring sensors, and the rotating speed is obtained by the time interval between the adjacent two arrival of a blade. Then the measurement error of method presented with no OPR sensor is analyzed, and experiments are conducted. The results indicate that the preprocess method presented with no OPR has better accuracy compared with the method with OPR sensor, so it is feasible to be used in the vibration parameter analysis.

\section{THE REPROCESSING METHOD WITH OPR SENSOR}

The existing BTT method uses OPR signal as the reference of timing, as is shown in Figure 1. The OPR sensor is mounted near the shaft and triggers when it detects a mark on the shaft. For arbitrary blade $j, \gamma_{j}$ is the angle between the blade and the mark on the shaft, $\theta_{i}$ is the angle between the OPR sensor and the tip-timing sensor $i$, and the two angles are calibrated before the measurement. So the angle between sensor $i$ and blade $j$ in no vibration can be calculated when the OPR signal triggers.

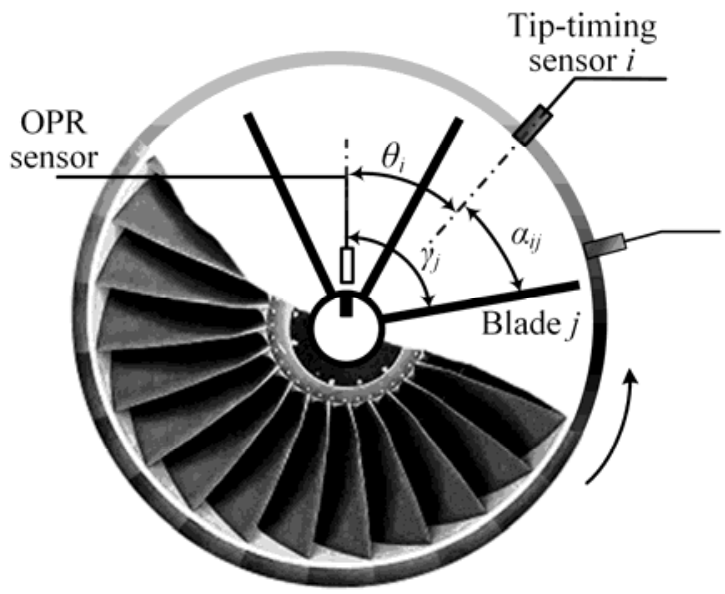

FIGURE I. TYPICAL BTT METHOD

The sensor begins to time after the OPR signal triggers in each circle. The time of arrival(TOA) $t_{i j}$ of the blade $j$ is 
measured, and the circumferential angle $\alpha_{i j}^{\prime}$ is calculated with the rotating speed, then the vibration displacement $y$ is

$$
y=R \alpha_{i j}^{\prime}-R \alpha_{i j}=R \int_{0}^{t_{i j}} \Omega(t) d t-R \alpha_{i j},
$$

where $\mathrm{R}$ is the radius of the rotor, $\Omega$ is the rotating speed. The rotating speed is calculated by the time interval between the two adjacent OPR signals in constant speed operation. So the measured blade vibration displacement is

$$
y=R \alpha_{i j}^{\prime}-R \alpha_{i j}=R \Omega t_{i j}-R \alpha_{i j}
$$

Abbreviate $\alpha_{i j}$ in (2) as $\alpha, t_{i j}$ as $t$. Analyze the measurement error of vibration displacement, and the result is

$$
\Delta(y) \approx \frac{\partial y}{\partial R} \Delta(R)+\frac{\partial y}{\partial \Omega} \Delta(\Omega)+\frac{\partial y}{\partial t} \Delta(t)+\frac{\partial y}{\partial \alpha} \Delta(\alpha) .
$$

The primary influence factors of blade vibration displacement measurement include four variables: radius of the rotor, rotating speed, the installation angle of the sensor and the tip-timing data. The radius of the rotor is usually much larger compared with the radius change of the rotor during the working process, and in some cases only the angular displacement of the blade vibration is of interest. So the effect of the radius of rotor is usually negligible, so (3) can be simplified as

$$
\Delta(y) / R \approx t \Delta(\Omega)+\Omega \Delta(t)-\Delta(\alpha)
$$

The error of blade vibration displacement measurement can be expressed as (4) when angular displacement is the major consideration. The arrival time change caused by blade vibration is very small, so $t \approx \alpha / \Omega$. Equation (4) is

$$
\Delta(y) / R \approx \alpha \frac{\Delta(\Omega)}{\Omega}+\Omega \Delta(t)-\Delta(\alpha)
$$

The angle between the sensor probe and the blade $\alpha$ equals to $\gamma_{j}-\theta_{i}$ as in Figure 1 , and $\gamma_{j}$ and $\theta_{i}$ both use the circumferential position of OPR sensor as reference, so the circumferential position of OPR sensor is the reference of the $\alpha$. Obviously, this reference is not in the same axial position as the blades and tip-timing sensors and might be affected by the bend and torsion vibration of the rotating shaft, which will introduce measurement error to the result of vibration displacement. And the error increases with the axial distance between the OPR sensor and the rotor.

According to (5), the effect of the rotating speed measurement error on vibration displacement measurement increases with the angle between the sensor and the blade when the OPR signal triggers. When the OPR signal triggers there will always be some blades whose angle $\alpha$ in Eq. 1 is close to $2 \pi$, and the vibration displacement measurement error caused by rotating speed error will reach the maximum. The accuracy of vibration displacement measurement can be improved by adding an angle encoded sensor which subdivide the shaft. But it increases the system complexity and the angle encoded sensor is hard to install, especially in multi-stage rotor engines. As the analysis above, the vibration displacement measurement method which use OPR signal as reference have some disadvantages in accuracy and installment. Hence a preprocessing method for BTT which use no OPR sensor is presented.

\section{The Preprocessing Method Without OPR SENSOR}

Traditional BTT method use OPR signal as time reference to calculate the arrival time of the blade in no vibration. In the condition OPR signal is unavailable, the vibration information can be obtained by calculating the difference of the TOA between two tip-timing sensors.

Assume there are $K+1$ tip-timing sensors installed around the rotor numbered $\# 0 \sim K$ respectively. The installing angle of sensor $\# 0$ is 0 , and $\Delta \alpha_{k}$ is the installing angle of sensor $\# k$, which is defined as the angle between sensor \#k and sensor \#0. Assuming that when the blade arrives at sensor $\# 0$, the blade vibration displacement is $y_{0}$, and the blade vibration displacement is $y_{k}$ when it arrives at sensor $\# k$. So

$$
y_{k}+R \Omega t_{k}-y_{0}=R \Delta \alpha_{k}
$$

$R$ is the radius of the rotor, $\Omega$ is the rotating speed and $t_{k}$ is the time of the blade rotating from sensor \#0 to sensor \#k. Let $t_{0 k}$ be the time of the blade rotating from sensor $\# 0$ to sensor $\# k$ when the blade is not in vibration.

$$
t_{0 k}=\Delta \alpha_{k} / \Omega,
$$

so (6) is

$$
\Delta y_{k}=y_{k}-y_{0}=R \Omega\left(t_{0 k}-t_{k}\right)=R \Delta \alpha_{k}-R \Omega t_{k} .
$$

$\Delta y_{k}$ is the vibration displacement difference of the blade between the two sensors, and it also contains the information of the blade vibration. The $\Delta y_{k}$ can be used in the subsequent algorithms to identify the blade vibration parameter. So the measurement accuracy of the vibration displacement difference $\Delta y_{k}$ is critical to the vibration analysis methods without OPR sensor.

According to (7) and (8), the primary influence factors of blade vibration displacement difference measurement include radius of the rotor, rotating speed, the installation angle of the sensor and the tip-timing data. Similar to the analysis above, the measurement error of the vibration displacement difference can be expressed as

$$
\Delta\left(\Delta y_{k}\right) / R=\Delta\left(\Delta \alpha_{k}\right)-\Delta \alpha_{k} \frac{\Delta(\Omega)}{\Omega}-\Omega \Delta\left(t_{k}\right)
$$


The angle $\Delta \alpha_{k}$ use the installation position of the tip-timing sensor as reference. The sensors and the rotor are in the same axial position. The error introduced by the bend and torsion vibration of the shaft is much smaller compare with the method with OPR sensor. The angle between two sensors is fixed during the measurement, so the impact of the measurement error of rotating speed is the same to all blades, according to (9). Compared with the method use OPR sensor, the impact of the measurement error of rotating speed can be reduced effectively by limiting the installation angle between the sensors. And the error introduced by any other noise of OPR sensor can be eliminated.

\section{EXPERIMENTS}

Experiments are conducted on a test rig to validate the signal preprocessing methods presented. A rotor with 16 blades is utilized and the radius of the rotor is $95 \mathrm{~mm}$. Two optical fiber based tip-timing sensors and an OPR sensor are used in the experiment, as Figure 2 shows. The rotating speed is $900 \mathrm{rpm}$, and the blade vibration displacement is small at a low speed. The installation position error of the sensors can be considered as a constant at the low speed. So the standard deviations of the tip-timing value are mainly related to the error of rotating speed.

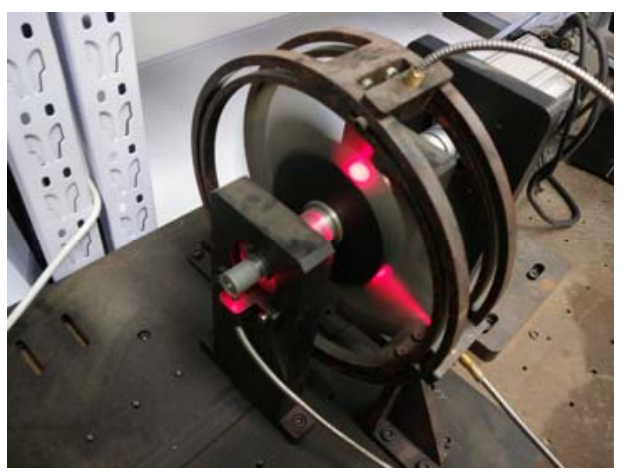

FIGURE II. PHOTO OF THE TEST RIG

Figure 3 and Figure 4 show the measured vibration displacement value of tip-timing signal in 1000 circles with DC component removed. Figure 3 shows the vibration displacements of blade $\# 0$ and blade $\# 8$, which is processed with the traditional method using the OPR sensor. The results in Figure 4 are processed with the method presented. The standard deviations of different blades measured with the two methods are presented in Figure 5.

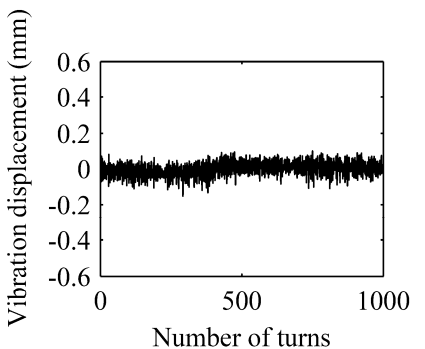

(A)

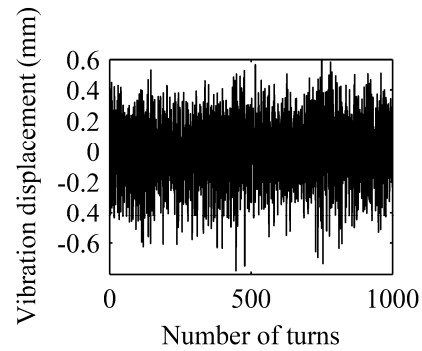

(B)
FIGURE III. THE PREPROCESSED RESULT OF THE METHOD WITH OPR SENSOR. (A) IS THE RESULT OF BLADE \#0 AND (B) IS THE RESULT OF BLADE \#8

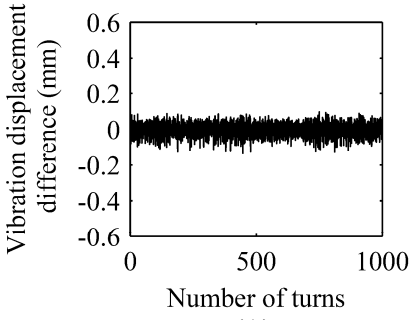

(A)

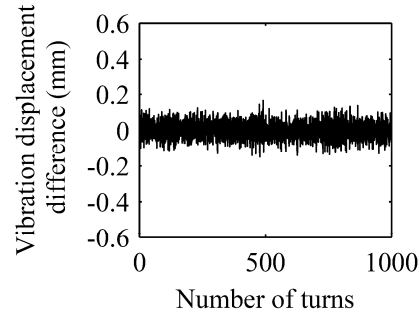

(B)
FIGURE IV. THE PREPROCESSED RESULT OF THE METHOD WITH NO OPR SENSOR WHEN THE ANGLE BETWEEN THE TWO SENSORS IS $8.6^{\circ}$. (A) IS THE RESULT OF BLADE \#0 AND (B) IS THE RESULT OF BLADE \#8

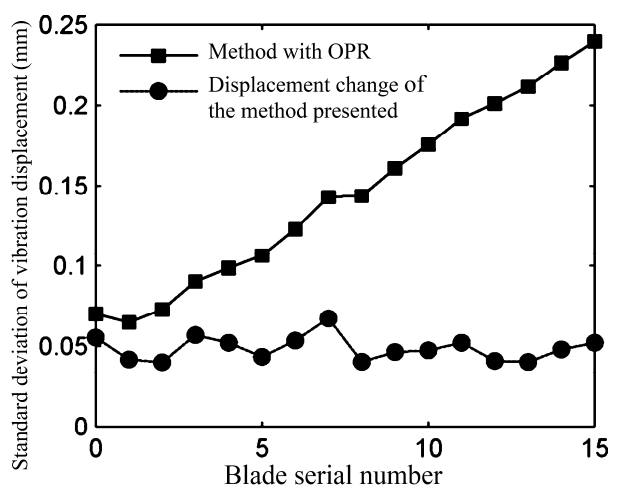

FIGURE V. THE STANDARD DEVIATIONS OF THE RESULTS PROCESSED WITH THE TWO METHOD

The experiment was conducted with the angle between the two sensors changed from $8.6^{\circ}$ to $90.4^{\circ}$, and the position of the first sensor is fixed. Figure 6(a) and Figure 5(b) show the vibration displacement difference of blade $\# 0$ and blade $\# 8$ measured with no OPR signal respectively, and compared to Figure 4, the standard deviations will be larger.

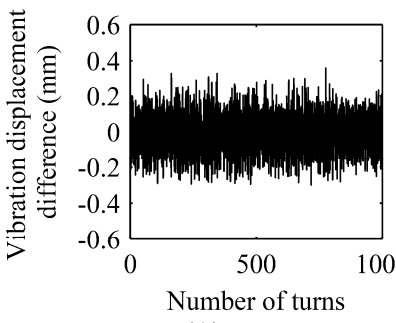

(A)

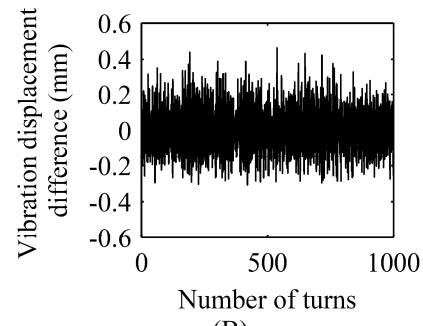

(B)
FIGURE VI. THE PREPROCESSED RESULT OF THE TWO METHODS WHEN THE ANGLE BETWEEN THE TWO SENSORS IS 90.4

As is shown in the experiment, the error of the vibration displacement measured with the method based on the OPR sensor is different for each blade. The error increase with the blade serial number, because the angle between the blade and the sensor is larger for the blade with big serial number when the OPR signal arrives. However, the error of the vibration displacement difference measured with the method without OPR is nearly the same for different blades, and the error is usually smaller when the installation angle between the sensors is relatively small. The error increases with the angle between the two sensors, and the difference is not much between 
different blades. So the experimental results are coincident with the theoretical analysis.

Simulation is conducted to analyze the effect of the SNR of the preprocessed tip-timing data with the two methods on vibration parameter identification. Two methods based on the single degree of freedom motion model are utilized in the simulation. The method with OPR uses the single parameter method, and the method without OPR is similar to the single parameter method. In the simulation, the natural frequency of the blade is $1800 \mathrm{~Hz}$, the damping coefficient is 0.0008 , the static displacement caused by the exciting force is $0.1 \mu \mathrm{m}$, the initial phase is $\pi / 6$, and the rotating speed varies from $170 \mathrm{~Hz}$ to $190 \mathrm{~Hz}$. The engine order is 10 , and the angle between the two sensors in the method with no OPR is $0.15 \mathrm{rad}$. White Gaussian noise of different amplitude is added to the signal. As is shown in Figure 3, the amplitude of vibration displacement of blade \#8 with OPR is approximately three times that of the vibration displacement difference with no OPR, which means approximately $9.5 \mathrm{db}$ lower in SNR. And this value is used in the simulation though the errors of blade \#9 to blade \#15 are bigger. Figure 7(a) shows the fitting result of the vibration displacement signal when the SNR is $20.5 \mathrm{db}$. Figure $7(\mathrm{~b})$ shows the fitting result of the vibration displacement difference signal when the SNR is $30 \mathrm{db}$.
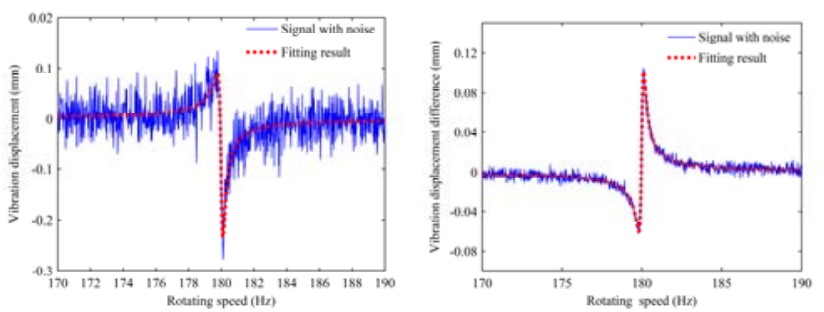

FIGURE VII. FITTING RESULT OF SINGLE PARAMETER METHOD (A)WITH THE OPR SIGNAL (B) WITH NO OPR SIGNAL

Vibration parameter identification is conducted 30 times and the standard deviation of the relative errors of the two methods are listed in Table I for comparison. As is shown in Table I, the SNR of simulative signal have obvious effect on the relative error of parameter fitting, the higher SNR of the method with no OPR improves the accuracy of vibration parameter identification. And when the traditional method with OPR signal fails to identify the vibration parameter accurately due to excessive noise, as the last two lines in Table I shows, the method with no OPR can still work because of the advantage in SNR. The simulation result indicates that the preprocessing method presented with no OPR can improve the accuracy of the vibration parameter analysis based on BTT.

TABLE I. RELATIVE ERROR OF PARAMETER FITTING

\begin{tabular}{cccccc}
\hline \hline SNR & Method & Amplitude(\%) & $\begin{array}{c}\text { Nature Frequency } \\
\text { (\%) }\end{array}$ & $\begin{array}{c}\text { Damping } \\
\text { Coefficient } \\
(\mathbf{\%})\end{array}$ & $\begin{array}{c}\text { Phase } \\
\text { (\%) }\end{array}$ \\
\hline $30 \mathrm{db}$ & With OPR & 0.90 & $1.2361 \times 10^{-3}$ & 1.58 & 2.51 \\
$30 \mathrm{db}$ & With no OPR & 0.91 & $1.1970 \times 10^{-3}$ & 1.15 & 1.82 \\
$20.5 \mathrm{db}$ & With OPR & 5.19 & $4.9413 \times 10^{-3}$ & 5.91 & 8.38 \\
$20 \mathrm{db}$ & With no OPR & 2.68 & $3.9428 \times 10^{-3}$ & 3.58 & 6.90 \\
$10.5 \mathrm{db}$ & With OPR & 18.256 & 12.32 & 10329.18 & 57.74 \\
\hline \hline
\end{tabular}

\section{CONCLUSION}

In this article, a signal preprocessing method for Blade tiptiming is analyzed. The method requires no OPR sensor which makes it more applicable in some cases the OPR sensor is difficult to install. The accuracy of the preprocessed data is critical to ensure the accurate identification of the vibration parameter through the following identification algorithm. The measurement errors of both the method presented and the typical preprocessing method with the OPR sensor are analyzed, and experiments are conducted to verify the methods.

The preprocess method with no OPR sensor is less affected by the shaft bend and vibration. The impact of rotating speed measurement on the error of vibration displacement difference is more stable and can be reduced by limiting the installation angle between the sensors. The theoretical analysis and experiments indicate that the signal preprocess method presented has a better accuracy compared with the method with OPR, so it provides a good foundation for the identification algorithm without OPR sensor. The method can also be used as a supplement of the BTT method with OPR sensor to reduce the error introduced by rotating speed measurement, or used to analyze acquired data when the OPR sensor fails to work properly.

\section{REFERENCES}

[1] S. Kumar, N. Roy and R. Ganguli, "Monitoring low cycle fatigue damage in turbine blade using vibration characteristics," Mech Syst Signal Pr, vol. 21, no. 1, pp. 480-501, Jan.2007.

[2] W. Lucjan, "Experimental crack propagation and failure analysis of the first stage compressor blade subjected to vibration," Eng Fail Anal, vol. 16, no. 7, pp. 2163-2170, Oct. 2009.

[3] A. R. Rao and B. K. Dutta, "Vibration analysis for detecting failure of compressor blade", Eng Fail Anal, vol. 25, pp. 211-218, Oct.2012.

[4] D. H. Diamond, P. S. Heyns and A. J. Oberholster, "A comparison between three blade tip Timing algorithms for estimating synchronous turbomachine blade vibration," in Proc. WCEAM, 2015, pp. 215-225.

[5] J. Gallego-Garrido, G. Dimitriadis, J. R. Wright, "A Class of methods for the analysis of blade tip-timing data from bladed assemblies undergoing simultaneous resonance. Part 1: theoretical development," International Journal of Rotating Machinery, article ID 27247, 2007.

[6] G. Dimitriadis, B. Carrington, J. R. Wright and J. E. Cooper, "Blade tip timing measurement of synchronous vibrations of rotating bladed assemblies," Mech Syst Signal Pr, vol. 16, no. 4, pp. 599-622.

[7] G. Iker, B. Josu, Z. Joseba, A. Gotzon, A. I. María and J. Felipe, “An optical fiber bundle sensor for tip clearance and tip timing measurements 
in a turbine rig," Sensors, vol.13, no. 6, pp. 1047-1058, June.2013.

[8] P. Procházka and F. Vaněk, "New methods of noncontact sensing of blade vibrations and deflections in turbomachinery," IEEE Trans. Instrum. Meas, vol. 63, no. 6, pp. 1583-1592, June.2014.

[9] C. P. Lawson and P. C. Ivey, "Tubomachinery blade vibration amplitude measurement through tip timing with capacitance tip clearance probes," Sensor Actuat A-Phys, vol. 38, no. 1, pp. 14-24, Jan.2005.

[10] M. Violetti, Q. Xu, O. Hochreutiner and A. K. Skrivervik, "New microwave sensor for on-line blade tip timing in gas and steam turbines," in Proc. APMC, 2012, pp. 1055-1057. 